\title{
Revision surgery for chiari malformation decompression
}

\author{
Catherine A. Mazzola, M.D., and Arno H. Fried, M.D. \\ Department of Neurosurgery, Pediatric Neurosciences Institute, Hackensack University Medical \\ Center, Hackensack, New Jersey
}

\begin{abstract}
Chiari malformations comprise four different hindbrain anomalies originally described by Hans Chiari, a professor of pathology at the German University in Prague. There are four basic Chiari malformations. The reasons for revision of Chiari malformation decompression may be for conservative or inadequate initial decompression or the development of postoperative complications. Another reason involves cases of both hindbrain herniation and syringomyelia in patients who have undergone adequate posterior fossa decompression without resolution of symptoms, signs, or radiological appearance of their syrinx cavity. Additionally, symptom recurrence has been reported in association with various types of dural grafts. Reoperation or revision surgery for patients with Chiari malformations is common and may not be due to technical error or inadequate decompression. The types of revision surgeries, their indications, and initial presentations will be reviewed.
\end{abstract}

KEY WORDS • Chiari malformation • revision surgery • decompression • complication

Chiari malformations, as described by Chiari in $1891,{ }^{3}$ represent four different congenital hindbrain anomalies: malformations of the brainstem, cerebellum, and/or the posterior fossa, or skull base itself. The cerebellar tonsils are caudally displaced through the foramen magnum in the Chiari Type I malformation. There may also be quadrigeminal plate abnormalities and/or syringomyelia. The Chiari Type II malformation is similar, as described by Cleland, ${ }^{4}$ but it is the cerebellar vermis that is usually herniated inferiorly within the foramen magnum. In patients with Chiari Type II malformation, the fourth ventricle is abnormally long, the tectum or collicular plate is beaked, the central canal of the spinal cord may be dilated, and the outlet of the foramen of Magendie may be covered by scar tissue, adhesions, or even by a thick membrane. ${ }^{4}$ Chiari Types III and IV malformations are rare and will, therefore, not be discussed in great detail. Chiari III malformations are described as a posterior fossa encephalocele that contains brainstem and cerebellar tissue within the sac. This unfortunate condition presents in utero or at birth, and the affected infants are severely compromised. The neural tissue within the encephalocele is usually gliotic and dysfunctional. These lesions are completely different from true high cervical myelomeningoceles and/or meningoceles; in children presenting with the latter, prognosis is usually good. Chiari IV malformations are a form of

Abbreviations used in this paper: $\mathrm{CMJ}=$ cervicomedullary junction; $\mathrm{CSF}=$ cerebrospinal fluid; $\mathrm{CT}=$ computerized tomography; DREZ = dorsal root entry zone; $\mathrm{ICP}=$ intracranial pressure; $\mathrm{MR}=$ magnetic resonance; $\mathrm{VP}=$ ventriculoperitoneal. cerebellar hypoplasia and should not be thought of as a "hindbrain herniation." Patients harboring these lesions exhibit surprisingly little neurological dysfunction, and the condition may be found in an asymptomatic patient.

\section{CHIARI I MALFORMATION}

Patients with Chiari I malformation may present as children or as adults. The detection of Chiari I malformation, especially in asymptomatic patients, has increased since the advent and widespread use of MR imaging for the evaluation of headaches. Patients with Chiari I malformation may be asymptomatic. Symptoms, when present, range from mild headache to severe myelopathy and/ or brainstem-related syndromes. Pain has been reported as the most frequent complaint in many clinical series; headache is also a common complaint. Pain is usually described in the occipital or cervical location and may increase with the Valsalva maneuver. Nonradicular pain may also be noted. Motor, sensory, cerebellar, and cranial nerve dysfunction may also be observed in older patients. Diplopia, dysarthria, and dysphagia may be noted by the patient. Even sinus bradycardia, coughing spasms, and hiccups have been described as presenting complaints of patients with Chiari I malformation. Greenlee, et al., ${ }^{9}$ reported a series of 31 pediatric patients who presented with Chiari I tonsillar herniation before the age of 6 years. Their chief complaints included oropharyngeal dysfunction in $35 \%$, scoliosis in $23 \%$, headache or neck pain in $23 \%$, sensory disturbances $(6 \%)$, weakness $(3 \%)$, and other symptoms. ${ }^{9}$ Signs of Chiari I malformation are relat- 
ed to the area of compression; if the cerebellar tonsils are primarily involved, the patient may present with signs related to cerebellar compromise and/or dural irritation (Lhermitte sign). If there is pressure on the posterior aspect of the cervical cord, one may find hypo- or hyperreflexia, or a Babinski sign. Oscillopia, vertical nystagmus, vocal cord dysfunction, abnormal gag reflex, and other cranial nerve findings are rare in Chiari I malformation, unless there is significant herniation or brainstem compression. Children and adults may also present with scoliosis as their only symptom; neurological status may be normal or abnormal. Note that papilledema, if present, may be indicative of another underlying pathological process such as pseudotumor cerebri or true hydrocephalus. These patients may not benefit from a simple Chiari decompression, and ICP monitoring or VP shunt placement may be warranted.

\section{CHIARI II MALFORMATION}

The medulla, pons, and the entire fourth ventricle may be involved in the caudal displacement in patients with Chiari II malformation. Vascular structures, which may also be displaced and are of surgical concern, are the posterior inferior cerebral arteries and the basilar artery. Chiari II malformation is strongly associated with spina bifida aperta and is seen in infants with myelomeningocele and myeloschisis. The reported incidence of hydrocephalus in infants with spina bifida aperta is approximately 90 to $95 \%$. Infants with Chiari II malformation present with unique symptoms directly related to congenital brainstem malformation and cranial nuclei abnormalities, as well as cervicomedullary compression. These symptoms may consist of stridor and/or aspiration due to vocal cord paresis or paralysis, apnea and breath-holding spells, failure to thrive, hypotonia, and quadriparesis. Older children and adults with a known diagnosis of spina bifida and hydrocephalus must undergo careful follow up. Many of these patients harbor a concomitant Chiari II malformation herniation that may become acutely symptomatic. Patients with Chiari II malformation and shunt-related dysfunction may present with symptoms of acute herniation. ${ }^{23}$ For several reasons, shunt-treated cases with Chiari II are often difficult to manage. In these patients with preexisting hindbrain herniation, a shunt malfunction might become rapidly symptomatic and fatal if not treated. Even adult patients with spina bifida and shunt-treated hydrocephalus, who suffer headache or neck pain, should undergo careful CT scanning, funduscopy to rule out papilledema, and follow-up evaluation to ensure proper shunt functioning. In the series reported by Lee, et al., ${ }^{15} 41 \%$ of children with Chiari II malformation and shunt malfunction presented with neck pain, $12 \%$ with lower cranial nerve palsy, and $12 \%$ with symptoms related to development of a syrinx. Four of 17 children with Chiari II malformation and shunt malfunction presented with seizure. In a child with Chiari II malformation and shunt malfunction, MR imaging demonstrated injury of the medial part of the inferior cerebellum and the medulla oblongata. ${ }^{23}$

Despite adequate decompression, brainstem symptoms may persist; such patients tend to have a poor prognosis. This may be due to the concomitant abnormalities of the intrinsic brainstem nuclei, as proposed by Charney, et al. ${ }^{2}$ Reevaluation to determine the adequacy of decompression is nonetheless warranted, because these unfortunate infants do develop tight bands and arachnoid adhesions that can cause recurrent stenosis and CMJ compression. ${ }^{21}$ Teo, et al., ${ }^{21}$ have described a system of grading useful for determining the severity of clinical, radiological, and operative findings in patients with Chiari II malformation. Magnetic resonance images of the craniocervical junction should be obtained in all three planes (coronal, sagittal and axial) to evaluate the CSF signal around the medulla within the foramen magnum. If there is no CSF signal around the cord at the level of the foramen magnum, or if a restrictive band of fibrous tissue is apparent, then decompression should be undertaken. In the series of 30 Chiari II malformation patients reported on by Teo, et al., ${ }^{21} 10$ required reoperation for recurrent compression and resultant symptoms.

On preoperative MR images, it is important to note the position of the torcula and transverse sinus. The transverse sinus and the torcula are often inferiorly displaced. This can make the initial suboccipital craniectomy quite challenging.

\section{CHIARI MALFORMATION DECOMPRESSION SURGERY}

Chiari malformation decompression surgery has three main goals: 1) decompression of the inferior aspect of the cerebellum; 2) enlargement of the total volume of the posterior fossa; and 3) establishment of CSF flow. These goals are independent of each other, in that the volume of the posterior fossa may be adequately increased without sufficient cerebellar decompression or establishment of CSF flow. In patients with Chiari malformation and syringomyelia, neurosurgeons may opt to decompress the syrinx at the first surgery or to perform the suboccipital decompression and follow the syrinx conservatively by using MR imaging. There have been no Class III studies in which investigators have reported a significant difference in outcome between one approach and the other. Myelotomy or syringotomy at the time of surgery may be warranted. Additionally, placement of a stent to drain the syrinx at the time of the first surgery may be considered. The benefits and drawbacks of syringotomy tubes will be discussed later.

Each step of the decompressive surgery can be analyzed with regard to its significance, effect, and related complications. To evaluate the various complications/conditions requiring reoperation, it is helpful to first review the various steps of the procedure. These aspects include: 1) positioning of the patient; 2) incision; 3 ) craniotomy or craniectomy; 4) dural opening; 5) decompression; 6) resection of arachnoid adhesions; 7) removal of inferior tonsils; 8) opening of the obex; 9) syrinx decompression (if desired); 10) dural closure; 11) closure of the craniotomy; and 12) fascial and skin closure (note that not all of these steps are always necessary).

In standard suboccipital approaches; the midline surgical incision is made from the inion to approximately C-3. The incision is brought down through the skin and midline nuchal fascia. To minimize blood loss, the avascular plane 
of the ligamentum nuchae is incised using a sharp scalpel or electrocautery with a blunt or needle tip. Care is taken not to penetrate the dura prematurely. Once the suboccipital bone and the $\mathrm{C}-1$ and $\mathrm{C}-2$ laminae have been carefully exposed, osseous decompression is performed. The craniotomy or craniectomy may be undertaken using a Hudson brace and a large Leksell rongeur; alternatively, one may use a high-powered drill with an acorn or burr (for the burr holes) and a cutting bit with a footplate (for the craniotomy). There are many ways to perform the craniotomy. Some surgeons prefer making two burr holes, below and on either side of the torcula, whereas others prefer drilling with a fine burr directly below the torcula. In any case, attention must be paid to avoiding excessive blood loss. Bone wax and thrombin-soaked Gelfoam are quite useful in packing off vascular channels within a thick occipital bone, as the craniotomy is performed. Again, in patients with Chiari II malformation the lowlying torcula and transverse sinus may make the suboccipital craniectomy difficult. Care should be taken when removing the posterior foramen magnum, especially if the transverse sinus and torcula lie just above the foramen. Once the craniotomy is finished, one must decide whether to leave out the bone. Many surgeons prefer a simple craniectomy to remove any aspect of osseous compression that may be involved. Enough bone should be resected to provide adequate access to the cerebellar tonsils and the obex below. The $\mathrm{C}-1$ arch is typically removed using either Kerrison rongeur, Leksell rougeur, or a drill.

Once hemostasis has been attained and the incision irrigated clean, the dura may be opened. Some surgeons do not open the dura, opting to perform an osseous decompression. It is our practice to open the dura, usually in a standard "Y"-shaped fashion. The dural opening is usually started at the crotch of the "Y" and continued laterally and upward, to complete the arms of the "Y." It is often helpful to clamp across the occipital sinus, with fine hemostats, prior to cutting across the sinus, between the two hemostats. The sinus can then be ligated using No. 4-0 nonabsorbable suture. This avoids clip-related artifacts on MR imaging studies. Again, note the position of a lowlying sinus in patients with Chiari II malformation. The dura may be temporarily or permanently secured to the adjacent lateral fascia for better exposure of the contents of the inferior posterior fossa.

Once the dura is retracted laterally, lysis of arachnoid adhesions and resection of the cerebellar tonsils may be safely performed. Many neurosurgeons undertake these next steps of the decompression with the assistance of the operating microscope. Arachnoid bands or adhesions may be cut sharply by using microscissors or a microdissector. The inferior cerebellar tonsils may either be removed using cavitating ultrasonic aspiration, or they may be shrunk using bipolar coagulation.

The obex may be checked to determine if it is open and if CSF flows easily from the fourth ventricle. Gardner ${ }^{8}$ has described "plugging" of the obex, with muscle or fascia, to obliterate pulsations from the fourth ventricle transmitted to the central canal.

A shunt may be placed between the fourth ventricle and the subarachnoid space if there is concern about maintaining an open CSF conduit. The drawback of placing a small piece of tubing in the foramen of Magendie, however, is that the tubing may become displaced or cause the formation of arachnoid adhesions. It is our practice to remove any adhesions blocking the outlets from the fourth ventricle at the time of the first decompression. If adhesions block the obex, careful dissection and release may also allow CSF flow from the fourth ventricle into and around the central canal. If reobstruction of the outlets of the fourth ventricle occurs postoperatively and the patient presents without papilledema but with a dilated fourth ventricle, then shunting of the fourth ventricle to the subarachnoid space may be considered. On occasion, however, thick bands of adhesions may develop in patients with Chiari II malformation. These adhesions may be too tenacious to lyse or they may damage normal tissue if not removed. Care should be taken when dissecting these adhesions; one should not become overly zealous at the expense of damaging normal tissue.

Decompression of a syrinx may be considered at the time of the first surgery; however, it is our practice not to perform a syringotomy at the time of the initial surgery. Several authors have reported that suboccipital craniectomy with duraplasty, in most patients, will be sufficient treatment and that the syrinx will resolve after the initial decompression. ${ }^{6,16}$ Simple dorsal midline myelotomy or DREZ myelotomy, however, can be performed at the time of the initial procedure. Opening the syrinx to drain its contents or placement of a stent to maintain the CSF conduit are acceptable means to decompress a syrinx. There are various stents available: syringosubarachnoid, syringopleural, and syringoperitoneal.

Once this is finished, the surgeon decides whether to close the dura or leave it open. If the dura is left open, the muscle and fascia must be carefully closed in two watertight layers. If the dura is closed, many surgeons prefer to use a dural graft to increase the intracranial volume of the posterior fossa. Various auto- and allografts have been used. Rosen, et al., ${ }^{19}$ reported complications related to using bovine pericardium, Gore-Tex, and lyophilized cadaver dura. They recommended using autologous grafts obtained from the pericranium. Other autologous sources of dural graft material include ligamentum nuchae and fascia lata. Certainly, however, there are benefits and drawbacks to using any of the various dural grafts. The dural graft is usually sewn into place. Duragen is a dural substitute that may be simply laid down over the defect. Fibrin glue may be used in the dural closure. The bone is replaced, in some cases, with suture, miniplates, or other cranial fixation devices. In other cases, the bone is left out.

The fascia is closed using absorbable suture, and the skin is closed using absorbable or nonabsorbable suture. Surgical staples may also be used for skin closure. Standard dressings such as Telfa and gauze are usually applied. Alternatively, Dermabond may be used as a bioocclusive dressing.

\section{Complications Related to Conservative Surgery (Persistent Compression)}

Complications related to conservative surgery may occur for a variety of reasons and include the following: 1) inadequate craniectomy; 2) persistent occipitoatlantal membrane; 3 ) inadequate dural opening/duraplasty; 4) re- 
sidual arachnoid adhesions; and 5) residual tonsillar compression.

Postoperatively, patients improve symptomatically; however, in a subgroup of patients, symptoms may recur several weeks or even months after surgery. Fessler and Rhoton $^{7}$ reported their experience at the University of Florida in Gainesville in 1992. When recurrent symptoms develop or if symptoms persist, MR imaging of the brain and cervical spine is performed. Persistent compression of the cerebellar tonsils may be apparent on sagittal and axial images, with inadequate CSF spaces around the inferior aspect of the cerebellum. This may be in part due to inadequate osseous decompression, persistent occipitoatlantal membrane, or an excessively tight, or restenotic, dural closure. If symptoms are mild, the patient may be followed conservatively. If clear compression exists, however, reoperation should be considered with extension of the decompressed region. In young children, for example, bone growth at the edges of the craniectomy or complete reclosure of a craniotomy may occur. If the dura was primarily closed at the time of the first surgery, placement of a dural graft should be considered at the time of revision surgery.

Persistent arachnoid adhesions due to scarring, which may have occurred before or even after surgery, can also cause irritation of the inferior cerebellar tonsils. Wide lysis of adhesions at the time of reoperation is necessary in this case.

Large swollen cerebellar tonsils may also cause symptomatic compression. In this case, tonsillar resection or bipolar coagulation of the superficial arachnoid over the inferior tonsils may help open CSF pathways and relieve compression.

\section{Postoperative Complications}

The following complications related to surgery can occur and may present early or late, depending on the cause and effect: 1) operative site hemorrhage; 2) aseptic meningitis; 3 ) inflammatory reaction involving the dural graft; 4) CSF leak and pseudomeningocele; 5) hydrocephalus; 6) wound infection; 7) large craniectomy and/or duraplasty and cerebellar sag; and 8) other craniotomy complications such as infection and displacement.

Operative site hemorrhage is rare but can occur in the parenchyma of the cerebellum, the subdural space, or epidural space. Hemorrhage is usually benign and self-limited, but any patient with hemorrhage-induced neurological deficit has to undergo neuroimaging and careful evaluation for evacuation of the hematoma if it should enlarge.

Aseptic meningitis is not a complication of surgery per se but may occur after an uneventful decompression. Children or adults may complain of severe headache, nausea, vomiting, lethargy, or other symptoms. Neither CT nor MR imaging is usually diagnostic, but examination of images can rule out other causes of symptoms. Irritation of the fourth ventricle and its surrounding structures may cause transient symptoms that usually improve after a short course of intravenous hydration, antiemetics, headache medication, and occasionally steroid agents. Rarely does aseptic meningitis last more than a few days; it does not require reoperation.

Rosen, et al., ${ }^{19}$ have reported on outcomes in three pa- tients who underwent suboccipital decompression and duraplasty. ${ }^{19}$ In each patient, a different graft was used. All three developed postoperative recurrence of symptoms 4 to 18 months after initial surgery. Recurrent compression was demonstrated on MR imaging. At surgery scarring, inflammation, and partial calcification of the grafts were noted. The neurosurgeons believed that the recurrent symptomatology was directly related to the graft used in each case. They contended that the use of autologous graft material may prevent inflammatory reactions, which were seen in their three patients.

Cerebrospinal fluid leak, pseudomeningocele, hydrocephalus, and wound infection can present as both early and late complications. Inadequate dural closure or gaps within the fascial closure may allow CSF to leak from the posterior fossa. Additionally, postoperative hydrocephalus, for any reason, is probably the most common cause of CSF leakage and wound breakdown, with or without pseudomeningocele formation. There have been reports of acute hydrocephalus following suboccipital decompression for Chiari I malformations resulting from large infratentorial supracerebellar hygromas. ${ }^{5}$ Large CSF collections in the subdural spaces and even pseudomeningoceles can cause CSF flow obstruction and hydrocephalus. Temporary ventricular drainage may obviate the need for insertion of a permanent shunt. Hydrocephalus that recurs after removal of an external ventricular drain may require placement of a shunt.

If CSF leakage is observed, a sterile dressing may be temporarily placed over the site. The leakage site along the suture line, however, should be closed using a figureeight stitch. The patient should undergo MR imaging to evaluate ventricle size and the occipitocervical area. If the ventricles are enlarged or if papilledema exists, a temporary ventricular drain may be placed. If leakage continues or resumes after the drain is removed, and if ventriculomegaly persists, then shunt insertion should be considered. If leakage has occurred and a shunt is indicated, one should consider a lumbar puncture to rule out the possibility of an occult CSF infection prior to shunt surgery.

The development of a pseudomeningocele is not necessarily a problem unless there is CSF leakage, papilledema, persistent syringomyelia, or if the cosmetic defect is unacceptable to the patient. As in the aforementioned scenario, it is important to conduct neuroimaging and ophthalmological examination to rule out ventriculomegaly and elevated ICP. If the ventricles have not enlarged and there is no elevation of ICP, then the pseudomeningocele can be followed conservatively. Pare and Batzdorf ${ }^{17}$ have reported an association between persistent syringomyelia and pseudomeningocele formation after Chiari malformation decompression. They described three patients in whom syringomyelia resolved only after their postoperative pseudomeningoceles were repaired. If hydrocephalus and syrinx are absent, Diamox (acetazolamide) may be used to decrease CSF production. If the pseudomeningocele is substantial, however, or if the cosmetic defect is unacceptable, then revision of the closure can be undertaken. The closure should be reopened down to the level of the dura. If the dura was intentionally left open, it may then be closed and a duraplasty performed using auto- or allograft. Synthetic dural substitutes may also be placed, with or 
without fibrin glue. Attention should be paid to prevention of CSF leakage at the time of dural reconstruction. Gelfoam or Duragen is placed over the entire duraplasty or along the edges as needed. The fascia should be then closed in multiple layers. There are no reports indicating any significant relation between the type of suture closure and the prevention of CSF leakage. Some surgeons prefer using a running locked suture line; others prefer a running nonlocked line; and still others prefer interrupted plain or interrupted figure-eight closure. Relatively heavy, absorbable suture should be used to close the fascia. To reclose the skin, many surgeons advocate the use of nylon nonabsorbable suture. If there is no evidence of infection, however, absorbable suture may be used to close the skin. Dermabond as an additional bioocclusive dressing may be helpful.

Wound infections are particularly problematic. Superficial wound infections may be treated with oral or intravenous antibiotic agents, topical cleansing and antibiotic ointments, and debridement if necessary. If there are areas of clear necrosis, they should be excised and reclosed. If the fascia underlying the infection is intact, there is less concern about meningitis. Deep wound infections, however, may cause bacterial meningitis. In cases of questionable or deep wound infections, a lumbar puncture should be performed to evaluate CSF gram stain and cultures to rule out occult infection. Any meningitis should be appropriately treated. Deep infections are quite rare; osteomyelitis requires removal of the infected bone and longterm intravenous antibiotic therapy.

Another rare postoperative complication is cerebellar sag due to an over zealous decompression. Patients may present weeks or months after surgery with persistent or new complaints related to brainstem and/or cerebellar displacement that has caused cervicomedullary compression. An excessive increase in the posterior fossa volume may allow the cerebellum and brainstem to herniate or sag into the area of decompression. Sagittal MR imaging will demonstrate the extent of displacement. There are two treatment options for cerebellar sagging. Revision surgery with partial reconstruction of the posterior fossa can be accomplished by a supportive cranioplasty. In patients with symptoms and signs of elevated ICP, the syndrome may also be managed appropriately by the placement of a VP shunt. In rare cases, both strategies may be needed to correct the symptomatic cerebellar herniation. We have treated one patient with pseudotumor cerebri, who presented with a lumbopleural shunt already in place. The patient had done well for a few months after lumbopleural shunt surgery. A few months later, she developed occipital headaches, attributed to a previously "undiagnosed" Chiari I malformation. She underwent decompression of the malformation and again did well for a few months.

The acquisition of Chiari I malformation tonsillar herniation has been reported in patients with various syndromes causing intracranial hypertension. ${ }^{11}$ In patients with craniofacial syndromes, hydrocephalus and pseudotumor cerebri Chiari I-related symptoms may develop with VP shunt dysfunction or excessive drainage from a working lumboperitoneal shunt. There has even been case reports of Chiari herniation after lumbar puncture. ${ }^{11}$ In patients with lumboperitoneal shunts, replacing the lum- bar shunt with a ventricular shunt may be the best treatment. In patients with acquired Chiari I herniation, if treated with suboccipital decompression alone, further caudal displacement may develop, exacerbating their symptoms. Our aforementioned patient, in fact, underwent a suboccipital decompression and then developed severe neck pain and sensory dysfunction a few months later. Her MR imaging studies revealed significant cerebellar sagging and caudal displacement (Fig. 1 left). On CT scanning, crowding within the foramen magnum and obliteration of CSF spaces were evident (Fig. 1 center). After careful discussion with the patient, we chose to place a programmable VP shunt (Fig. 1 right). The valve was set at its lowest setting. The working lumbopleural shunt was removed 2 months later. Her symptoms resolved within 1 month after placement of the VP shunt.

\section{Other Complications After Adequate Decompression}

The following complications have been associated with Chiari decompression surgery: persistent cervical syringomyelia, occipitocervical instability, anterior basilar invagination, arachnoid cyst development/arachnoid scarring, reformation of the posterior atlantooccipital membrane, and new or persistent symptoms despite negative imaging studies.

Patients who present with large or holocord syringomyelia and Chiari I herniation are challenging to treat. Management is confounded by the decision of whether to decompress the syrinx at the time of the original surgery or to undertake a standard suboccipital decompression and duraplasty.

Feldstein and Choudri ${ }^{6}$ reported on seven pediatric patients who presented with findings of holocord syringes and Chiari tonsillar herniation. These patients underwent suboccipital decompression and duraplasty. Significant collapse of the syrinx cavities was demonstrated in six patients; in one a $50 \%$ collapse of the syrinx was observed. Magnetic resonance imaging was performed at 2 to 4 months and at 1 year after surgery. All patients improved clinically, even though one patient experienced persistent syringomyelia.

Persistent syringomyelia can occur even after excellent suboccipital decompression. Munshi, et al., ${ }^{16}$ reported an increased rate of syrinx resolution when suboccipital decompression is combined with duraplasty. They found that all of nine of their patients with Chiari malformation I and a syrinx who underwent duraplasty had a postoperative decrease in syrinx size; this was in contrast to three of six in whom the syrinx decreased in size after suboccipital craniectomy alone. If imaging studies demonstrate adequate decompression but persistent syrinx after surgery, the patient may undergo conservative follow-up evaluation. If there are associated symptoms or scoliosis, MR imaging should be repeated with and without administration of intravenous contrast within 3 to 6 months to ensure the absence of occult spinal disease, such as a neoplasm. If the syrinx persists and is symptomatic then syringotomy and drainage, with or without placement of a shunt, should be considered. Small silicone stents have been placed into syrinx cavities as syringosubarachnoid shunts. These stents are sometimes difficult to position and secure to the arachnoid; however, No. 6-0 or 8-0 Pro- 


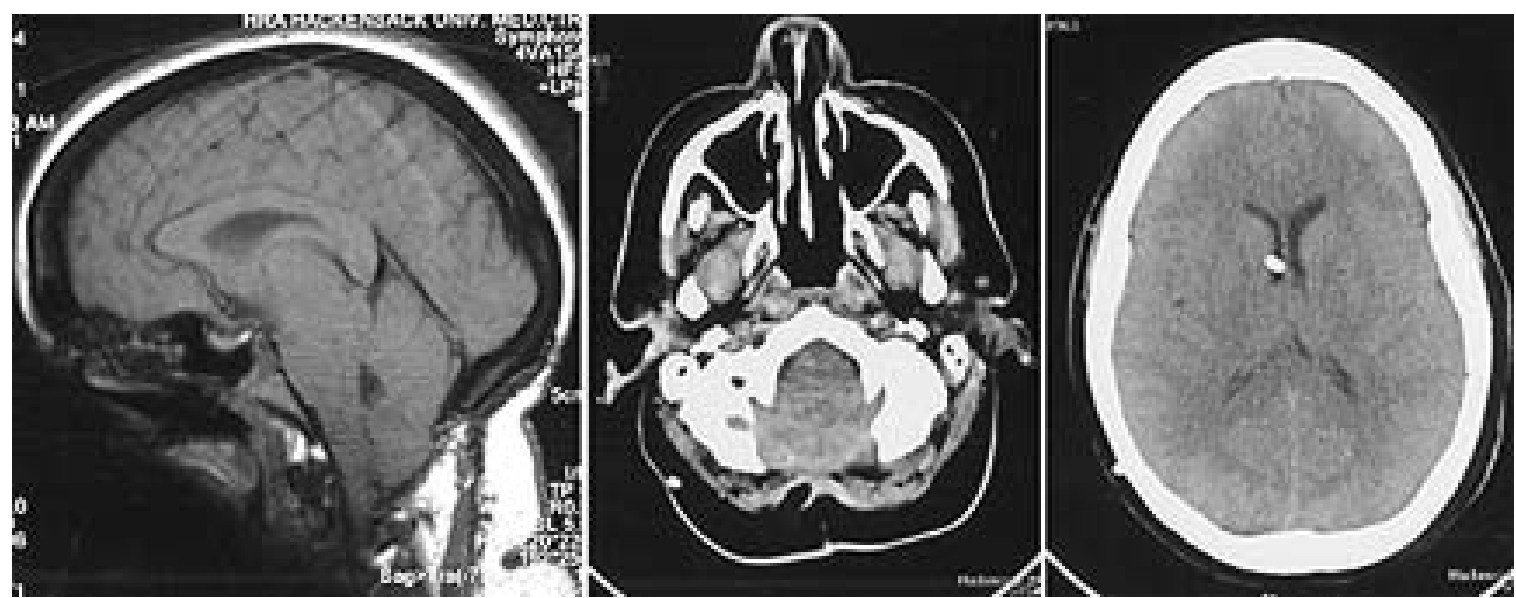

Fig. 1. Neuroimaging studies obtained in a patient who underwent suboccipital decompression for Chiari herniation following placement of a lumboperitoneal shunt for pseudotumor cerebri. Left: Sagittal MR imaging revealing caudal displacement of cerebellar tonsils into area of decompression (cerebellar sagging). Center: Axial CT scan demonstrating crowding of the brainstem and cerebellar structures within the area of the decompression. Note reduction of CSF spaces. Right: Axial CT scan revealing the placement of a right ventricular catheter into the frontal horn of the lateral ventricle. A programmable VP shunt was placed.

lene has been used to attach these small stents in position. Similarly, small T-shaped tubes have been placed to drain the syrinx into the subarachnoid spaces. Iwasaki, et al., ${ }^{13}$ reported their extensive experience with syringosubarachnoid shunt placement. They found that in patients who underwent DREZ myelotomy and placement of the shunt into the anterolateral subarachnoid space, reoperation was unnecessary. This finding was in contrast to that in patients who underwent midline or DREZ myelotomies and placement of the shunt into the posterolateral or posterior subarachnoid spaces. Syringopleural and syringoperitoneal shunts have also been used to divert CSF from within the syrinx; however, proximal placement is difficult, and migration and/or occlusion of either the proximal or distal tubing may occur. Damage to the perilesional spinal cord may also occur. Only in clear cases of syrinx expansion or in cases in which symptoms persist despite adequate decompression should aggressive syrinx decompression be undertaken.

Occipitocervical instability may develop in a delayed fashion in patients who have undergone Chiari malformation decompression. Instability may be due to congenital osseous deformities or ligamentous weakness. If an overly zealous suboccipital craniectomy is performed or if the C-1 and C-2 lamina are removed too widely, postoperative instability may develop. Patients may present with complaints related to pain or neurological dysfunction. Flexion-extension cervical radiographs and MR images may reveal subluxation or rotational instability. Rarely do these patients present with such severe instability; however, in extreme cases, patients may require occipitocervical stabilization. In such patients, fusion and stabilization are difficult because of the previous osseous decompression. In most Chiari malformation decompressions, the C-2 laminae does not need to be resected. By careful evaluation of preoperative MR images, the necessary extent of bone removal can be determined. In rare cases of severe Chiari II herniation, when the cerebellar vermis may herniate down as low as C-5, a suboccipital craniectomy and cervical osteoplastic laminotomy may be useful. Replacing the C-2, C-3, C-4, and C-5 laminae may help prevent postoperative cervical instability. The laminae may be secured using heavy absorbable suture in children; alternatively, miniplates, wires, or other fixation devices may be used.

Another cause of persistent or recurrent symptoms may be anterior basilar invagination and compression of the anterior CMJ. Patients with Chiari I malformation-related symptomatology should undergo careful preoperative assessment to determine if there is any component of anterior compression. If anterior compression is revealed, transoral decompression of the CMJ may successfully avert the need for suboccipital craniectomy and duraplasty. ${ }^{12}$ If anterior compression is found, alternatively, a posterior decompression combined with occipitocervical fusion may be considered. Fusion is performed to arrest progression of micromovement, which is believed to cause pannus or granulation tissue formation near the odontoid process.

The development of arachnoid cysts and spinal cord tethering after Chiari II decompression has been reported. ${ }^{10,19}$ Anteriorly situated arachnoid cysts have been discovered on postoperative MR imaging in patients with recurrent symptoms.$^{10}$ In some cases, brainstem compression caused new symptoms. Neck pain, vertigo, quadriparesis, extraocular muscle paresis, and upper-extremity dysmetria were symptoms associated with the anterior arachnoid cysts. In one study patients with arachnoid cysts underwent cyst fenestration or cystoperitoneal shunting. ${ }^{10}$ In another study the authors reported that the high cervical spinal cord was tethered to the dura by dense arachnoid adhesions..$^{20}$ In the case described by Takahashi, et al., ${ }^{20}$ the patient presented with neurological deterioration. Magnetic resonance imaging demonstrated new syringobulbia and scarring-induced obliteration of the posterior cervicomedullary CSF spaces. Patients with symptomatic tethering and syringobulbia due to adhesions and fi- 
brous scarring should undergo surgical release of the adhesions, placement of a syringosubarachnoid shunt, and duraplasty. ${ }^{20}$

The posterior atlantooccipital membrane is usually thickened at the time of the initial Chiari decompression. Typically the ligament is removed or opened prior to exposing the dura for the intracranial portion of the decompression; however, when a simple bone excision is performed, the ligament may be left intact. The posterior atlantooccipital membrane has been reported as a cause of recurrent symptoms after an initial period of improvement following a suboccipital craniectomy and decompression. ${ }^{22}$ In the patient reported on by Tubbs, et al., ${ }^{22}$ the posterior atlantooccipital membrane was initially opened and then reflected laterally to allow the dura to be opened. A duraplasty was performed with autologous graft obtained from the pericranium. Although initial results were good, at 3 months the patient experienced difficulty "clearing his secretions." Magnetic resonance imaging revealed an increase in syringomyelia and posterior fossa compression. At the time of reoperation, thickening and scarring of the posterior atlantooccipital membrane were observed. The dura was again opened, and a unilateral, tonsillar subpial coagulation was performed. At a 3-month follow-up visit, the child's symptoms and cervical syringomyelia had substantially improved. Teo, et al., ${ }^{21}$ have also described finding tight dural bands and adhesions that caused recurrent and primary compression in patients with Chiari II malformation.

Patients with persistent or even new symptoms should undergo reimaging postoperatively. If the CSF spaces around the posterior aspect of the cerebellar tonsils (or vermis in Chiari II malformation) are adequate and the fossa posterior decompression is good, then the cause of the symptoms may not be directly related to compression. Intrinsic pathological lesions within the brainstem, cerebellum, and/or spinal cord should be investigated. As a neurosurgeon, it is very frustrating to perform decompression with adequate results and then discover postoperatively that the patient's symptoms have not improved and that compression was not the cause. Nevertheless, in infants with Chiari II malformation, as previously mentioned, this is often the case. ${ }^{18}$ Whether due to an intrinsic cytopathological entity within the brainstem or to constricting fibrous bands at the occipitocervical junction (as part of the occipitoatlantal membrane), prognosis in these children is often poor. ${ }^{2,18}$ Additionally, pseudotumor cerebri and elevated ICP may present as a Chiari I syndrome with the neuroimaging findings consistent with cerebellar tonsillar herniation. When treated with a standard suboccipital decompression, symptoms in these patients may increase, likely related to cerebellar sagging, brainstem traction, and herniation. Decreased vision and increased papilledema may also occur in patients with pseudotumor cerebri in whom lumboperitoneal shunts are placed. ${ }^{14}$ Lancione and Kosmorsky ${ }^{14}$ have hypothesized that the development of Chiari-type herniation after placement of a lumbar shunt is due to increased ICP may be isolated to the ventricular system. Placement of a VP shunt in these patients is usually curative.

Bejjani, et al., ${ }^{1}$ reported on six patients with recurrent symptoms that developed 1.5 to 9 months after suboccip- ital decompression for Chiari I herniation. Postoperative MR imaging demonstrated adequate CSF signal and flow behind the cerebellar tonsils. Lumbar puncture was performed in all patients and ICP was mildly to significantly elevated. Patients underwent VP shunt placement or multiple lumbar punctures and acetazolamide therapy. They hypothesized that alterations in CSF hydrodynamics, inadequate initial decompression, or underlying intracranial hypertension may have existed in this subgroup of patients.

\section{CONCLUSIONS}

There are many reasons for revision surgery for Chiari malformation decompression surgery. Asymptomatic patients may present with imaging-documented findings consistent with persistent compression, whereas others may present with new or persistent symptoms related to residual compression. Too extensive a decompression may lead to extreme cerebellar herniation and sagging, with brainstem, spinal cord, or cerebellar symptoms. Aggressive osseous decompression may also lead to postoperative cervicospinal instability. Finally, there are "true" postoperative complications that may be due to errors in judgment, technique, or other natural causes. Even after "successful" surgeries, troublesome complications have developed, ranging from benign self-limited aseptic meningitis to severe wound infections and other conditions.

\section{References}

1. Bejjani GK, Cockerham KP, Rothfus WE, et al: Treatment of failed Adult Chiari Malformation decompression with CSF drainage: observations in six patients. Acta Neurochir 145: 107-116, 2003

2. Charney EB, Rorke LB, Sutton LN, et al: Management of Chiari II complications in infants with myelomeningocele. J Pediatr 111:364-371, 1987

3. Chiari H: Uber Veranderungen des Kleinhirns infolge von Hydrocephalie des Grosshirns. Dtsch Med Wschr 17: $1172-1175,1891$

4. Cleland J: Contribution to the study of spina bifida, encephalocele and anencephalus. J Anat Physiol 17:257-292, 1883

5. Elton S, Tubbs RS, Wellons JC III, et al: Acute hydrocephalus following a Chiari I decompression. Pediatr Neurosurg 36: 101-104, 2002

6. Feldstein NA, Choudhri TF: Management of Chiari I malformations with holocord syringohydromyelia. Pediatr Neurosurg 31:143-149, 1999

7. Fessler RG, Rhoton AL Jr: Chiari malformation and syringomyelia, in Little JR, Awad IA (eds): Reoperative Neurosurgery. Baltimore: Williams \& Wilkins, 1992, pp 113-127

8. Gardner WJ: Hydrodynamic mechanism of syringomyelia: its relationship to myelocele. J Neurol Neurosurg Psychiatry 28: 247-259, 1965

9. Greenlee JD, Donovan KA, Hasan DM, et al: Chiari I malformation in the very young child: the spectrum of presentations and experience in 31 children under age 6 years. Pediatrics 110:1212-1219, 2002

10. Jean WC, Keene CD, Haines SJ: Cervical arachnoid cysts after craniocervical decompression for Chiari II malformations: report of three cases. Neurosurgery 43:941-945, 1998

11. Johnston I, Jacobsen E, Besser M: The acquired Chiari malformation and syringomyelia following spinal CSF drainage: a study of incidence and management. Acta Neurochir 140: 417-428, 1998 


\section{A. Mazzola and A. H. Fried}

12. Kaibara T, Hurlbert RJ, Sutherland GR: Transoral resection of axial lesions augmented by intraoperative magnetic resonance imaging. Report of three cases. J Neurosurg (Spine 2) 95: 239-242, 2001

13. Iwasaki Y, Hida K, Koyanagi I, et al: Reevaluation of syringosubarachnoid shunt for syringomyelia with Chiari malformation. Neurosurgery 46:407-413, 2000

14. Lancione RR Jr, Kosmorsky GS: When does low mean high? Isolated cerebral ventricular increased intracranial pressure in a patient with Chiari I malformation. J Neuro-Ophthamol 21: 118-120, 2001

15. Lee TT, Uribe J, Ragheb, et al: Unique clinical presentation of pediatric shunt malfunction. Pediatr Neurosurg 30:122-126, 1999

16. Munshi I, Frim D, Stine-Reyes R, et al: Effects of posterior fossa decompression with and without duraplasty on Chiari malformation-associated hydromyelia. Neurosurgery 46: 1384-1390, 2000

17. Pare LS, Batzdorf U: Syringomyelia persistence after Chiari decompression as a result of pseudomeningocele formation: implications for syrinx pathognesis: report of three cases. Neurosurgery 43:945-948, 1998

18. Pollack IF, Kinnunen D, Albright AL: The effect of early craniocervical decompression on functional outcome in neonates and young infants with myelodysplasia and symptomatic Chiari II malformations: results from a prospective series. Neurosurgery 38:703-710, 1996
19. Rosen DS, Wollman R, Frim DM: Recurrence of symptoms after Chiari decompression and duraplasty with nonautologous graft material. Pediatr Neurosurg 38:186-190, 2003

20. Takahashi Y, Tajima Y, Ueno S, et al: Syringobulbia caused by delayed postoperative tethering of the cervical spinal corddelayed complication of foramen magnum decompression for Chiari malformation. Acta Neurochir 141:969-973, 1999

21. Teo C, Parker EC, Aureli S, et al: The Chiari II Malformation: a surgical series. Pediatr Neurosurg 27:223-229, 1997

22. Tubbs RS, Wellons JC III, Oakes WJ, et al: Reformation of the posterior atlanto-occipital membrane following posterior fossa decompression with subsequent constriction at the craniocervical junction. Pediatr Neurosurg 38:219-221, 2003

23. Yamada J, Morimoto K, Takemoto O: [Manifestation of Chiari II symptoms following peritoneal shunt tube extension.] No To Hattatsu 30:394-397, 1998 (Jpn)

Manuscript received June 24, 2003.

Accepted in final form August 22, 2003.

Address reprint requests to: Catherine A. Mazzola, M.D., Department of Neurosurgery, Pediatric Neurosciences Institute, Hackensack University Medical Center, 20 Prospect Avenue, Suite 800, Hackensack, New Jersey 07601. email: cmazzola@humed.com. 